\title{
XÂY DỰNG MÔ HİNH HỆ THỐNG ĐĂNG KÝ ĐẤT ĐAI ĐIỆN TƯ TRÊN ĐỊA BÀN TH!̣ XÃ TỪ SƠN, TỈNH BẮC NINH
}

\author{
TRỊH TH!̣ KIỀU TRANG, BÙI NGỌC TÚ, ĐOÀN QUANG CỦƠNG \\ Khoa Địa lý, Truờng Đại học Khoa học Tụ nhiên, ĐHQGHN
}

\section{Tóm tắt:}

Chính phủ Việt Nam nhấn mạnh tầm quan trọng của quản lý đất đai hiệu quả, bền vũng và cải thiện quản trị. Kể tù năm 2008, một số nỗ lục đã được thưc hiện nhung nhũng vuớng mắc co bản trong lĩnh vưc quản lý đất đai vẫn tồn tại, đặc biệt là trong việc xây dụng co sở dũ liệu và hệ thống đăng ký đất đai. Nghiên cứu cải thiện, xây dụng mô hình hệ thống đăng ký đất đai điện tử là một nghiên cứu cần thiết trong bối cảnh Chính phủ Việt Nam đang xây dụng hệ thống thông tin đất đai đa muc đích phù hợp với chiến lược phát triển Chính phủ điện tủ trong thời kỳ Cách mang công nghiệp 4.0. Nghiên cứu sủ dụng các phwơng pháp điều tra thu thập tài liệu, phân tích dũ liệu, phưong pháp thiết kế có cấu trúc, phwơng pháp lập trình web để đưa ra mô hình đăng ký đất đai điện tư phù hợp nhất. Kết quả nghiên cưu đã xây dưng được hệ thống đăng ký đất đai trục tuyến mới, góp phần nâng cao hiệu quả hệ thống quản lý đất đai đa muc tiêu cho Tù Sơn, Bắc Ninh và khả năng ứng dụng cho các địa phioong khác.

\section{Mở đầu}

Chính phủ Việt Nam nhấn mạnh vai trò của việc hoàn thiện hạ tầng thông tin đất đai ở Trung ương và địa phương đáp ứng mục tiêu xây dựng Chính phủ điện tử (CPĐT) và nâng cao hiệu quả quản lý đất đai. Ứng dụng công nghệ của Cách mạng công nghiệp 4.0 trong lĩnh vực quản lý đất đai góp phần thúc đẩy xây dựng và hoàn thiện cơ sở dữ liệu đất đai quốc gia tích hợp, gia tăng cơ hội cung cấp thông tin, chia sẻ dữ liệu, hỗ trợ ra quyết định trong lĩnh vực đất đai và phát triển các ứng dụng có liên quan.

Từ năm 2008, một số dự án phát triển hệ thống quản lý hồ sơ địa chính và cơ sở dữ liệu (CSDL) đã thực hiện với tổng vốn đầu tư hơn 380 triệu đô la. Các dự án đều nhấn mạnh mục tiêu hoàn thiện và vận hành hệ thống CSDL đất đai; hướng dần, cung cấp dịch vụ công thực hiện qua mạng điện tử trên toàn quốc, đặc biệt đối với đăng ký quyền sử dụng đất và các tài sản gắn liền với đất. Thực tế này đặt ra yêu cầu cần xây dựng một hệ thống CSDL và ĐKĐĐ có khả năng ứng dụng, triển khai rộng rãi hơn [6]. Tác giả Đỗ Thị Minh Tâm (2010) nghiên cứu thiết kế mô hình quản lý đất đai điện tử nhằm mục đích cải thiện khả năng cung cấp dịch vụ ở quy mô cấp huyện của thành phố Hà Nội đưa ra nhận định các dịch vụ liên quan đến đất đai, ĐKĐĐ, cấp GCNQSDĐ của Việt Nam còn chưa hiệu quả [5]. Olusoyi Ashaye (2012) xác định hệ thống quản lý đất đai điện tử là một hợp phần quan trọng trong $\mathrm{CPĐT} \mathrm{và} \mathrm{tính} \mathrm{hiệu} \mathrm{quả} \mathrm{của} \mathrm{CPĐT}$ được đánh giá thông qua khả năng cung cấp, chuyển đổi các dịch vụ và sự phù hợp với hệ thống quản lý đất đai - điều kiện tiên quyết cho gắn kết xã hội và phát triển kinh tế của các nước đang phát triển [4]. (Xem hình 1)

Thị xã Từ Sơn là khu vực phát triển nhanh và có nhiều biến động về đất đai, hệ thống thông tin đất đai được vận hành theo mô hình tập trung cấp tỉnh tuy nhiên chưa triển khai ĐKĐĐ trực tuyến (Hình 1) [8]. Nghiên cứu về cải thiện hệ thống ĐKĐĐ và xây dựng mô hình ĐKĐĐ điện tử trên địa bàn thị xã Từ Sơn, tỉnh Bắc Ninh là một nghiên cứu cần thiết trong bối cảnh chính phủ Việt Nam đang nỗ lực xây dựng một hệ thống thông tin đất đai đa mục đích.

\section{Dũ liệu và phương pháp nghiên cứu}

Ngày nhận bài: 05/09/2020, ngày chuyển phản biện: 09/09/2020, ngày chấp nhận phản biện: 15/09/2020, ngày chấp nhận đăng: 18/09/2020 


\subsection{Phương pháp điều tra, thu thập số liệu}

Điều tra số liệu thứ cấp: Các tài liệu, số liệu, bản đồ được thu thập tại Văn phòng Đăng ký đất đai (VPĐKĐĐ) chi nhánh Từ Sơn, Bắc Ninh.

Điều tra số liệu sơ cấp: sử dụng phương pháp điều tra nhanh với sự tham gia của cộng đồng và lấy ý kiến chuyên gia, kết quả phân tích thống kê từ 150 phiếu điều tra là cơ sở quan trọng khi thiết kế mô hình CSDL và ĐKĐĐ điện tử, cung cấp các dịch vụ phù hợp với yêu cầu kỹ thuật và đáp ứng nhu cầu thực tế.

\subsection{Phương pháp thiết kế có cấu trúc}

Phương pháp thiết kế có cấu trúc bằng ngôn ngữ mô hình hóa thống nhất (UML) cho phép mô hình hóa các ứng dụng của máy tính, thiết kế hệ thống thông tin dưới dạng các sơ đồ [1]. Nghiên cứu đã thiết kế hệ thống dưới dạng 03 loại sơ đồ: sơ đồ ca sử dụng thể hiện các chức năng và các tác nhân của hệ thống (Hình 2), các sơ đồ hoạt động để thể hiện các tiến trình hoạt động của hệ thống và sơ đồ lớp để thể hiện cấu trúc CSDL (Hình 3). Cấu trúc và mô hình cơ sở dữ liệu của hệ thống được xây dựng dựa theo Thông tư 75/2015/TT-BTNMT của Bộ Tài nguyên và Môi trường ban hành ngày 28/12/2015, quy định về nội dung CSDL đất đai. (Xem hinh 2, 3)

\subsection{Phuơng pháp lập trình Web}

Nghiên cứu xây dựng mô hình ĐKĐĐ điện tử theo giao diện web-based chạy trên mạng inter-

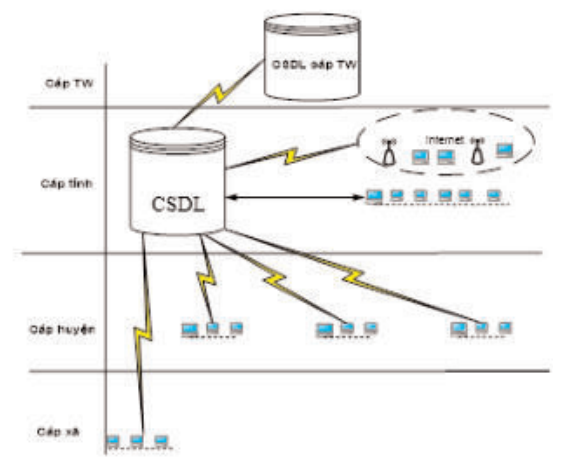

Hình 1: Mô hình vận hành hệ thống thông tin đất đai tập trung tại cấp tỉnh net. Từ bản thiết kế đã đề cập ở trên, các tác giả đã phát triển hệ thống bằng ngôn ngữ lập trình PHP (Hypertext Preprocessor) và JavaScript theo kiến trúc khách-chủ 3 tầng (3-tier clientserver architecture) thông thường [2, 3]. Các phần mềm được sử dụng để phát triển hệ thống hỗ trợ ĐKĐĐ gồm:

- Hệ quản trị CSDL PostgreSQL/PostGIS có vai trò quản lý CSDL không gian và thuộc tính;

- Dữ liệu bản đồ hiển thị trên giao diện Web được phát triển trên hệ thống pMapper để đơn giản hóa quá trình phát triển hệ thống và được quản lý bằng máy chủ bản đồ UMN MapServer.

Trong MapServer, các dữ liệu được hiển thị lên bản đồ thông qua việc thiết kế nội dung của file cấu hình gọi là Mapfile. Các chức năng của hệ thống được thiết kế dưới các dạng plugins của pMapper để người dùng sử dụng hệ thống một cách thuận tiện, dễ dàng. Việc xử lý số liệu trên máy chủ được thực hiện bằng ngôn ngữ lập trình PHP và tương tác với người sử dụng trên máy tính cá nhân bằng các đoạn mã JavaScript.

\subsection{Dũ liệu và khu vục nghiên cứu}

Từ Sơn là thị xã cửa ngõ phía Nam của tỉnh Bắc Ninh, có nhiều khu công nghiệp, làng nghề truyền thống nổi tiếng với quy mô 7 phường và 5 xã. Bắc Ninh là địa phương thử nghiệm dự án "Tăng cường quản lý đất đai và CSDL đất đai" (Vietnam Improved Land Governance and Database Project-VILG), hệ thống quản lý đất đai thuộc nhóm tiên tiến, hiện đại nhất trong cả

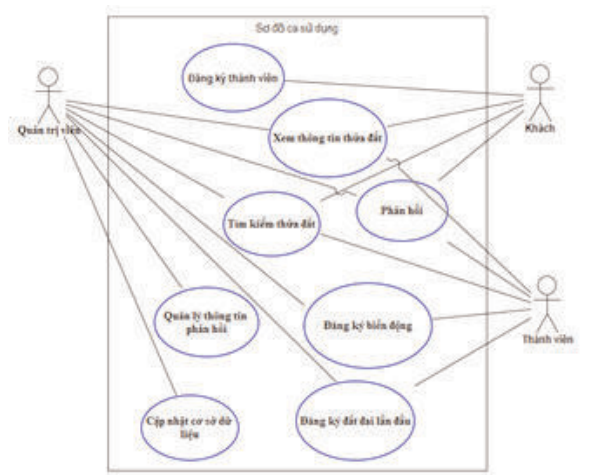

Hình 2: So đồ ca sư dụng của hệ thống 
nước. Việc ứng dụng công nghệ thông tin được UBND thị xã đặc biệt quan tâm, thực hiện công khai minh bạch và cập nhật đầy đủ các thủ tục hành chính trên website để người dân có thể theo dõi, kiểm tra. Trong năm 2019, chi nhánh VPĐKĐĐ thị xã Từ Sơn đã tiến hành thử nghiệm hệ thống thông tin đất đai đa mục tiêu (VietLIS) đáp ứng được những nhu cầu cơ bản trong quá trình cập nhật, lưu trữ và tra cứu CSDL đất đai trên địa bàn.

Mặc dù vậy, kết quả thực hiện nhiệm vụ quản lý đất đai và chất lượng dịch vụ ĐKĐĐ, cấp giấy chứng nhận quyền sử dụng đất, quyền sở hữu nhà ở và tài sản khác gắn kiền với đất (gọi tắt là Giấy chứng nhận quyền sử dụng đất GCNQSDĐ)...vẫn còn những hạn chế: quá trình chuyển đổi từ quản lý hồ sơ dạng giấy sang phần mềm VietLIS còn chậm, số lượng cán bộ biết sử dụng phần mềm VietLIS thấp (3/14 cán bộ được đi tập huấn sử dụng phần mềm VietLIS), tỷ lệ hoàn thành ĐKĐĐ lần đầu chỉ đạt $93,9 \%$, hơn 3.000 trường hợp chưa đủ điều kiện cấp GCNQSDĐ [7], người dân chưa thực sự hiểu rõ quy trình ĐKĐĐ và mất nhiều thời gian để hoàn thành hồ sơ, giấy tờ đăng ký... Do vậy, thị xã Từ Sơn được lựa chọn làm khu vực nghiên cứu, xây dựng mô hình hệ thống ĐKĐĐ điện tử và chạy thử nghiệm mô hình ở phường Đồng Nguyên. Các dữ liệu thu thập được trên địa bàn bao gồm:

- 52 mảnh bản đồ địa chính dạng số đã cập nhật biến động đến năm 2019.

- Các báo cáo kết quả thực hiện nhiệm vụ quản lý đất đai, báo cáo tình hình cấp GCNQSDĐ, đăng ký biến động đất đai, báo cáo đánh giá kết quả thực hiện nhiệm vụ năm 2019 trên địa bàn thị xã Từ Sơn.

- 150 phiếu điều tra thu thập ý kiến cá nhân, hộ gia đình và cán bộ quản lý đất đai ở xã, phường, VPĐKĐĐ đánh giá về thực trạng ĐKĐĐ và chất lượng dịch vụ, yêu cầu đối với hệ thống ĐKĐĐ trong tương lai...

\section{Kết quả thử nghiệm và thảo luận}

Để giúp cho công tác ĐKĐĐ, cấp GCNQSDĐ được thực hiện nhanh chóng, hiệu quả và công khai minh bạch thông tin đến các bên liên quan thì việc xây dựng CSDL đất đai là khâu quan trọng, cần phải thực hiện trước tiên.

Mô hình vận hành: Mô hình vận hành được đề xuất là CSDL đất đai được lưu trữ và vận hành tại 3 cấp Trung ương, tỉnh, huyện theo cơ chế kết nối liên thông, sao lưu dữ liệu ở 3 cấp. Mặc dù tăng chi phí đầu tư ban đầu nhưng về lâu

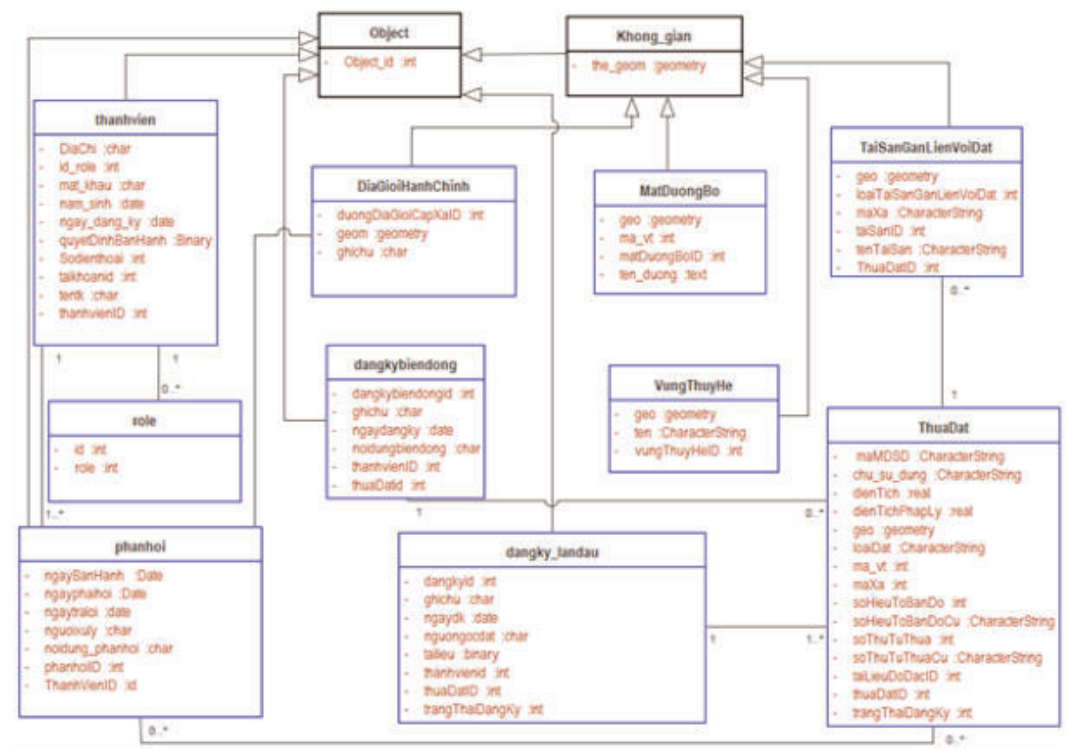

Hình 3: So đồ lớp thể hiện mô hình cơ sở dũ liệu của hệ thống 
dài hệ thống máy chủ 3 cấp mới đáp ứng đủ nhu cầu cập nhật, lưu trữ thông tin, tăng cường an ninh, bảo mật dữ liệu và cung cấp dịch vụ, thực hiện giao dịch điện tử thống nhất trên toàn quốc.

Hạ tầng kỹ thuật và hệ thống phần mềm nền: đầu tư thuê/mua hạ tầng kỹ thuật công nghệ thông tin đủ đáp ứng vận hành CSDL đất đai của các quận/huyện, tỉnh/thành phố, theo mô hình tập trung tại cấp Trung ương, phục vụ việc tác nghiệp hàng ngày của các VPĐKĐĐ truy cập trực tiếp vào hệ thống.

Đuờng truyền dĩ liệu: sử dụng hệ thống mạng nội bộ để đảm bảo sự ổn định và tính bảo mật vận hành hệ thống thông tin đất đai.

Phần mềm hệ thống thông tin đất đai: chuyển đổi các phần mềm khác nhau ở các địa phương thành một phần mềm thống nhất đảm bảo đáp ứng yêu cầu hệ thống, đưa vào vận hành cho hệ thống thông tin đất đai. Phần mềm phải đảm bảo các chức năng phục vụ tác nghiệp của cán bộ VPĐKĐĐ, hỗ trợ dịch vụ công, đảm bảo bản quyền và an ninh, bảo mật.

CSDL đất đai: đầu tư xây dựng CSLD đất đai từ cấp huyện, cập nhật sao lưu dữ liệu ở cấp tỉnh, kết nối các CSDL thành phần tại cấp Trung ương và các CSDL chuyên đề.

Từ dữ liệu bản đồ thu thập được, các tác giả đã thực hiện chuẩn hóa bản đồ bằng phần mềm Microstation. Sử dụng các công cụ của
Microstation để đưa các đối tượng về các lớp dữ liệu theo đúng quy định, kiểm tra các lỗi về quan hệ không gian (topology), sửa lỗi tự động, tạo vùng, đánh số thửa, vẽ nhãn thửa, kiểm tra thông tin thuộc tính của các thửa đất để đảm bảo mỗi thửa đất đều chứa đầy đủ thông tin thuộc tính bao gồm số hiệu thửa, loại đất, diện tích, chủ sử dụng, địa chỉ. Dữ liệu sau chuẩn hóa được đưa vào hệ quản trị CSDL.

Nghiên cứu đã xây dựng được một hệ thống WebGIS hỗ trợ ĐKĐĐ dưới dạng bản đồ trực tuyến. Sau khi người sử dụng đăng nhập, hệ thống có giao diện như trên Hình 4.

Các nhóm dữ liệu được xây dựng để phục vụ công tác ĐKĐĐ của phường Đồng Nguyên, thị xã Từ Sơn, tỉnh Bắc Ninh bao gồm: nhóm dữ liệu địa giới hành chính, nhóm dữ liệu giao thông, nhóm dữ liệu thủy hệ, nhóm dữ liệu thuộc tính của thửa đất, nhóm dữ liệu tài sản gắn liền với đất, nhóm lớp đăng ký lần đầu, nhóm lớp đăng ký biến động.

Mô hình xác định 3 nhóm đối tượng sử dụng hệ thống ĐKĐĐ trực tuyến bao gồm: khách, thành viên, quản trị viên. Chức năng, quyền hạn của các đối tượng sử dụng được trình bày trong Sơ đồ ca sử dụng (Hình 2). (Xem hình 4, 5)

Người sử dụng có thể tìm đến thửa đất được quan tâm bằng cách sử dụng các công cụ phóng to, thu nhỏ... và truy vấn thông tin về thửa đất

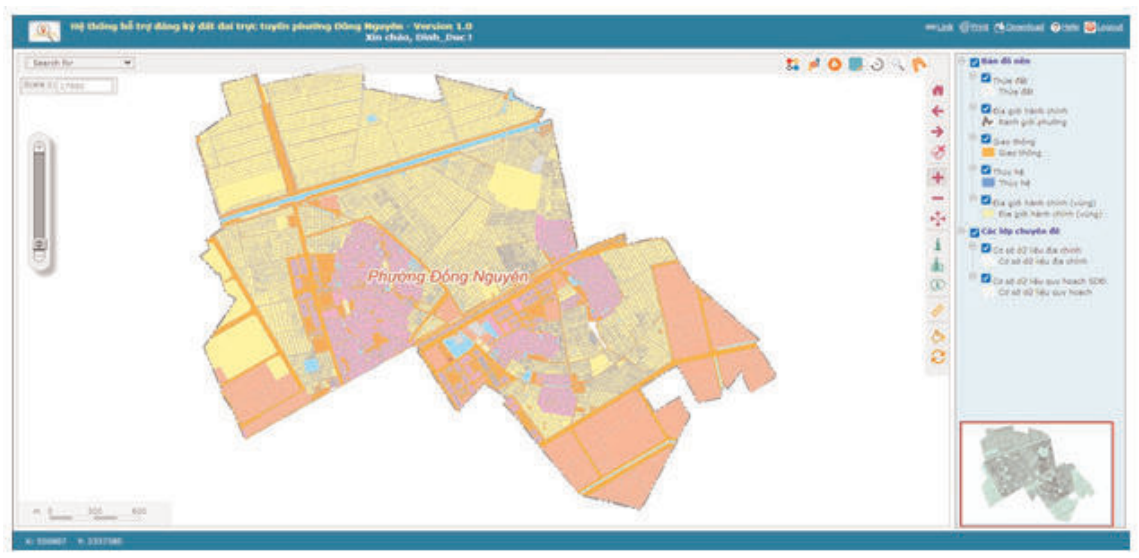

Hình 4: Giao diện trên hệ thống WebGIS sau khi đăng nhập 
bằng công cụ (xem cửa sổ ở góc dưới bên phải bản đồ Hình 5). Người dùng có thể tìm kiếm theo thuộc tính của đối tượng hoặc tìm kiếm theo vùng. Với chức năng này, người dùng có thể dễ dàng tìm kiếm thông tin về đối tượng quan tâm trong phạm vi vùng đệm. Kết quả tra cứu có thể được xuất thành file lưu hoặc in ấn dưới 3 định dạng word, excel, pdf.

Khi có nhu cầu ĐKĐĐ, cấp GCNQSDĐ, người sử dụng có thể thực hiện đăng ký bằng việc gửi các thông tin đăng $\mathrm{k} \square \mathrm{y}$ lên hệ thống qua các biểu mẫu được thiết kế sẵn. Có hai loại biểu mẫu chính trong quá trình thực hiện đăng ký là ĐKĐĐ lần đầu và đăng ký biến động đất đai. Đối với trường hợp thửa đất đã ĐKĐĐ lần đầu thì hệ thống sẽ hiển thị những thông tin được đăng ký và cho phép người sử dụng cập nhật đăng ký biến động (Hình 6). Sau khi thực hiện đăng ký, dữ liệu sẽ được cập nhật, hệ thống có thể hiển thị ngay tiến trình hồ sơ ĐKĐĐ đến giai đoạn nào. Tiến trình xử lý hồ sơ có thể được kết nối liên thông với cơ quan Thuế. Kết quả cuối cùng của $Đ K Đ Đ$ là GCNQSDĐ; các dữ liệu ĐKĐĐ được chiết suất dưới dạng Sổ Địa chính điện tử phục vụ cho công tác quản lý nhà nước về đất đai.

Người sử dụng có thể theo dõi và gửi thông tin trao đổi tới người quản trị hệ thống, tạo sự tương tác hai chiều giữa chính quyền và người dân trong giải quyết các vấn đề có liên quan. Nội dung phản hồi sẽ được hệ thống ghi nhận và gửi đến cán bộ quản lý. Công cụ còn hiển thị thời gian giải quyết các trao đổi của người sử dụng hệ thống. Với quản trị viên, các thông tin phản ánh có thể được xem bằng công cụ xem thông tin phản hồi (Hình 7, Hình 8).

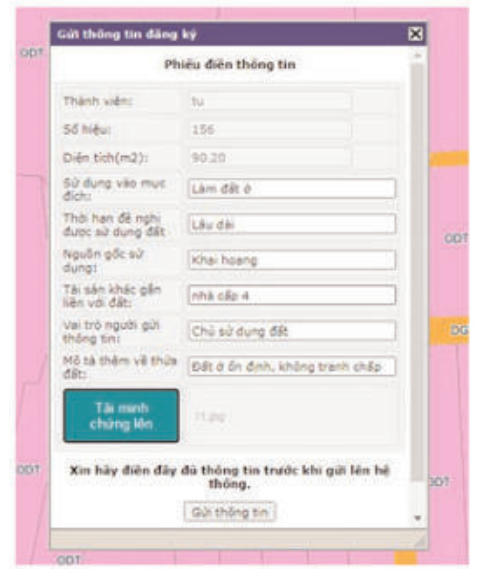

Hình 6: Giao diện chức năng gử thông tin đăng ký đất đai

Các cán bộ quản lý (quản trị viên) sẽ quản lý và kiểm soát các thành viên tham gia vào hệ thống cũng như phân quyền cho các nhóm thành viên. Trên cơ sở tiếp nhận các ý kiến đóng góp của người dân và các bên liên quan, cán bộ phụ trách sẽ tiếp thu những đóng góp, phản hồi và trả lời lại thông tin thông qua chức năng quản lý phản hồi.

Thông tin đăng ký của các thửa đất luôn được hệ thống cập nhật và quy chuẩn về thời điểm hiện thời nên hệ thống luôn đảm bảo cung cấp thông tin chính xác nhất đến người sử dụng. Như vậy, với chức năng kiểm tra tiến trình và

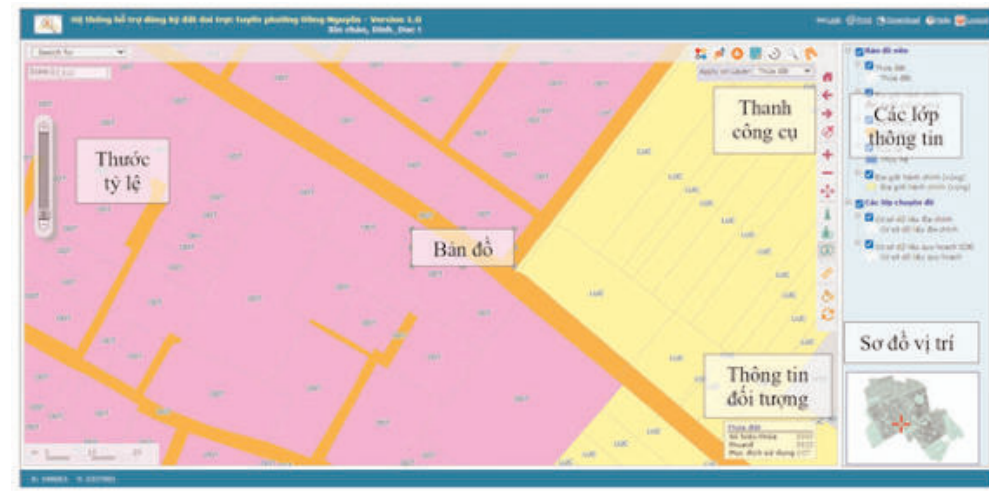

Hình 5: Giao diện chung của hệ thống với các chức năng được thiết kế duới dạng plug-in 


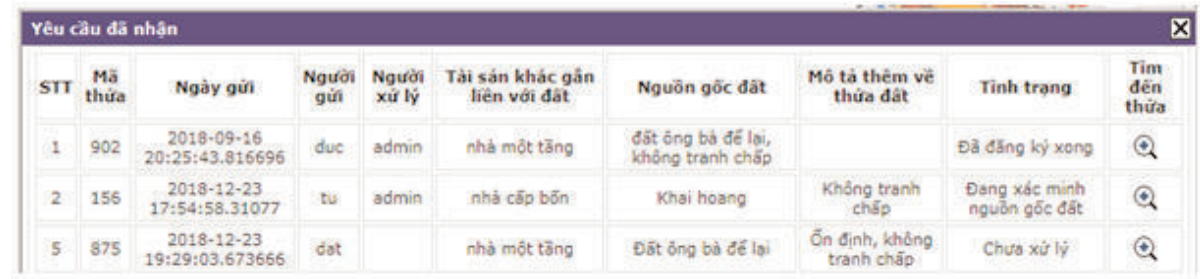

Hình 7: Theo dõi tiến trình đăng ký đất đai trên PostgreSQL

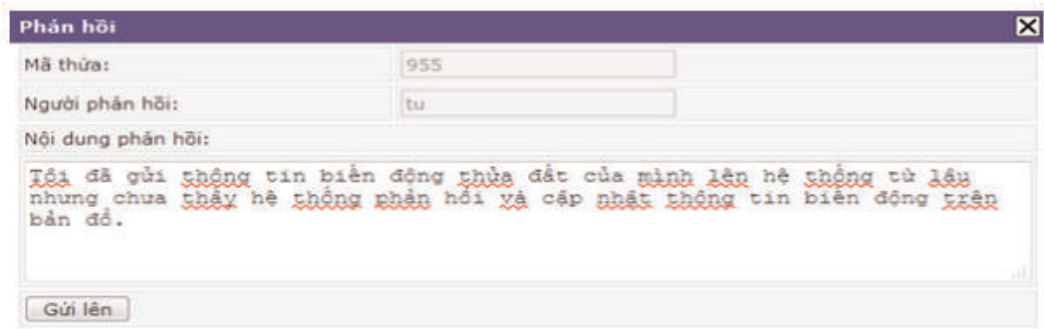

Hình 8: Ý kiến phản hồi của thành viên gử lên hệ thống

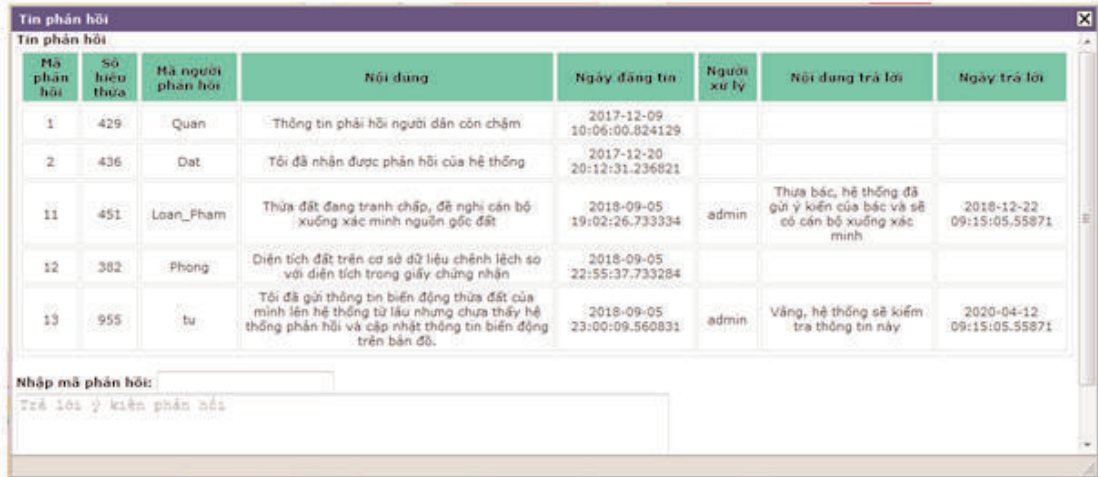

Hình 9: Quản lý phản hồi của cán bộ quản lý

xem thông tin phải hồi, người sử dụng gần như ngay lập tức biết được hồ sơ ĐKĐĐ đang được xử lý như thế nào, công khai và minh bạch. Tuy nhiên, hệ thống quản lý đất đai hiện nay chưa công nhận tính pháp lý của các văn bản điện tử và chữ ký điện tử trong các giao dịch, dịch vụ đất đai. Do vậy, cần có cơ chế mới và phù hợp với sự phát triển của hệ thống quản lý, ĐKĐĐ điện tử, sự kiểm duyệt thông tin và độ chính xác về mặt pháp lý của các thông tin, dữ liệu được gửi vào $\mathrm{CSDL}$ của hệ thống. Theo đề xuất của nghiên cứu, ở cấp vĩ mô, Trung ương cần sớm đi vào vận hành $\mathrm{CPĐT,} \mathrm{ban} \mathrm{hành} \mathrm{cơ} \mathrm{chế,} \mathrm{chính}$ sách đối với hoạt động hành chính điện tử như quản lý đất đai, ĐKĐĐ trực tuyến. Trong đó, yếu tố quan trọng đầu tiên là các quy định liên quan đến công nhận tính chất pháp lý của các giao dịch, dịch vụ công trực tuyến, chữ ký điện tử và giấy tờ có liên quan. Người quản lý hệ thống sẽ tiến hành kiểm tra, xác minh tính xác thực, mức độ tin cậy của các thông tin dựa trên tiêu chuẩn đã được ban hành. Sau đó, thông tin sẽ chính thức được đưa vào CSDL và cập nhật trạng thái hồ sơ ĐKĐĐ, bắt đầu quy trình đăng ký theo đúng thủ tục quy định. Trong trường hợp thửa đất đăng ký có tranh chấp, phức tạp trong xác minh thông tin trực tuyến thì có thể gửi phản hồi yêu cầu cung cấp thêm thông tin, minh chứng. 
Bảng 1: Kết quả khảo sát mức độ hài lòng đối với hệ thống

\begin{tabular}{|l|c|}
\hline \multicolumn{1}{|c|}{ Nội dung khảo sát } & Tỷ lệ đồng ý (\%) \\
\hline Thông tin đất đai được cung cấp tới từng thửa đất & 100 \\
\hline Chất lượng thủ tục ĐKĐĐ, quản lý thông tin của hệ thống & 86,7 \\
\hline Dễ dàng khi sử dụng chức năng của hệ thống & 90 \\
\hline Mức độ tương tác với người sử dụng & 90 \\
\hline Mong muốn hệ thống được triển khai trong thực tế & 100 \\
\hline
\end{tabular}

Như vậy, với cơ chế kiểm soát thông tin của hệ thống, người sử dụng vừa có thể tra cứu thông tin thửa đất từ trước đến nay ngay thời điểm hiện tại, đồng thời có thể tiến hành các thủ tục ĐKĐĐ và theo dõi tiến trình, kết quả chính thức từ hệ thống sau khi người quản lý kiểm duyệt và cập nhật thông tin.

Để đánh giá tính hiệu quả của hệ thống, nghiên cứu đã tiến hành khảo sát mức độ hài lòng của các bên liên quan về kết quả hệ thống mang lại. Đối tượng được lấy ý kiến là cán bộ tại chi nhánh VPĐKĐĐ thị xã Từ Sơn (3 người) và người dân địa phương (27 người). Các kết quả khảo sát được thống kê trong Bảng 1 .

Kết quả khảo sát cho thấy, hệ thống đã đạt được một số thành công nhất định. Tuy còn đơn giản và chưa thực hiện được tất cả các dịch vụ trong công tác quản lý đất đai nhưng nếu hoàn thành ĐKĐĐ với tỷ lệ $100 \%$ thì đây sẽ là CSDL quan trọng cho hệ thống quản lý minh bạch, hiệu quả. Tất cả đối tượng được hỏi đều đồng tình với cách thức hoạt động, thông tin hệ thống cung cấp và đều mong muốn một mô hình hệ thống tương tự có thể nhanh chóng được triển khai trong thực tế. Hệ thống hiện nay đã có đủ tính năng cơ bản đáp ứng nhu cầu tra cứu thông tin và thực hiện thủ tục đăng ký, tuy nhiên cơ chế kiểm duyệt thông tin và mức độ bảo mật cần tiếp tục hoàn thiện để đảm bảo chất lượng thực hiện thủ tục hành chính. Hướng phát triển tiếp theo của nghiên cứu là tích hợp CSDL đất đai từ hệ thống đăng ký hiện tại, kết nối với các hệ thống dữ liệu, các lớp thông tin chuyên đề khác để hoàn thiện hệ thống quản lý đất đai điện tử thống nhất trên cả nước.

\section{Kết luận}

ĐKĐĐ, cấp GCNQSDĐ là một nhu cầu tất yếu và đồng thời cũng là trách nhiệm của người sử dụng đất. Việc đáp ứng nhu cầu này một cách thuận tiện nhất sẽ giúp cho công tác quản lý và khai thác thông tin đất đai trở nên minh bạch và hiệu quả trong thời đại công nghệ 4.0.

Trên cơ sở ứng dụng công nghệ WebGIS, nghiên cứu đã xây dựng một hệ thống ĐKĐĐ điện tử trực tuyến đơn giản, hiệu quả, có tính tương tác cao với người sử dụng. Hệ thống có thể tiếp nhận và xử lý, cập nhật kết quả đăng ký trực tuyến theo từng thửa đất do người sử dụng hệ thống gửi đến. Cùng với đó, việc tra cứu thông tin đất đai và thông tin khác được thực hiện trên nền bản đồ địa chính giúp cho người dân trên địa bàn có thể tìm kiếm thông tin cần thiết một cách dễ dàng nhất. Hệ thống có thể được triển khai ở các địa bàn khác và ở quy mô lớn hơn trên cơ sở bổ sung thông tin vào CSDL và thiết kế hệ thống sao lưu, lưu trữ 3 cấp Trung ương, tỉnh và huyện.

Kết quả nghiên cứu cho thấy, công nghệ WebGIS và CSDL không gian có tính khả thi cao trong hỗ trợ công tác ĐKĐĐ và giải quyết các thủ tục hành chính về đất đai. Đồng thời hệ thống cũng góp phần cung cấp thông tin tới các bên có nhu cầu tìm hiểu thông tin thửa đất, xác minh nguồn gốc, tình trạng pháp lý hiện thời của thửa đất trước khi đưa ra các quyết định tài chính có liên quan. $\bigcirc$

\section{Lời cảm ơn}

Nghiên cúu này được tài trọ bởi Truòng Đại hoc Khoa họ Tụ nhiên trong đề tài mã số TN.19.11 


\section{Tài liệu tham khảo}

[1]. Trần Quốc Bình (2012), Giới thiệu về UML và Enterprise Architect, Trường ĐHKHTN, Hà Nội.

[2]. Pinde Fu and Jiulin (2012), WebGIS: Principles and Application, ESRI Press.

[3]. Lê Hoàng Sơn (2015), Lập trình ứng dụng WebGIS, Nhà xuất bản Giáo dục Việt Nam.

[4]. Olusoyi Ashaye (2012), Evaluating the Use of E-Government in the Implementation of Land Administration Systems in Developing countries: The case of in Negeria. Ph.D. dissertation, Brunel University, London.

[5]. Do Thi Minh Tam (2010), Designing egovernment based land administration system (e-LAS) for improving delivery of land adminis- tration services (Case study in Hanoi, Vietnam). International Institute for Geo-Information science and Earth Observation, Enschede, The Netherlands.

[6]. Trinh Thi Kieu Trang (2019), A Study on the Improvement of Land Registration System in Vietnam, Ph.D. dissertation. Daegu University, Korea. p. 82-83.

[7]. Báo cáo tổng hợp chi nhánh Văn phòng đăng ký đất đai thị xã Từ Sơn, tỉnh Bắc Ninh năm 2019.

[8]. Trung tâm Dũ liệu và Thông tin đất đai, Tổng cục Quản lý đất đai (2016), Báo cáo khảo sát dự án: Xây dựng hệ thống thông tin đất đai và Mô hình giao dịch đất đai điện tử, Hà Nội.O

\section{Summary}

\section{Development of e-land registration system model for Tu Son Town, Bac Ninh Province}

Trinh Thi Kieu Trang, Bui Ngoc Tu, Doan Quang Cuong

Faculty of Geography, VNU University of Science, 334 Nguyen Trai, Hanoi, Vietnam

The government of Vietnam highlights the importance of effective and sustainable land management and improved governance. Since 2008, some efforts have been undertaken to develop land records and databases. Despite much progress, fundamental bottlenecks in the land administration sector remain. There are still many shortcomings in the Vietnamese land administration system, especially in the land registration system. A study on the improvement of the land registration system is necessary in the context that the government of Vietnam is trying to build a multi-purpose land information system. The research purpose was to improve the efficiency and transparency of land administration services by developing the land registration system in Tu Son town, Bac Ninh Province. This research used survey methods, data analysis (qualitative analysis), statistical methods, comparison, and expert methods. This research results have proposed the new electronic land registration system that can improve the multi-purpose land management system for Tu Son, Bac Ninh in particular and in general in Vietnam. $O$ 